\title{
Novel Nano-Zirconium Antimonate as Cation Exchange Material for Organic Dye Pollutants Purification
}

\author{
M. F. Elkady 1, 2, *, M. A. khodary ${ }^{3}$, A. A. Zaatout ${ }^{3}$ \\ ${ }^{1}$ Chemical and Petrochemical Engineering Department, Egypt-Japan University of Science and Technology, New Borg El-Arab City, \\ Alexandria, Egypt \\ ${ }^{2}$ Fabrication Technology Department, Advanced Technology and New Materials and Research Institute (ATNMRI), City of Scientific Research \\ and Technological Applications, Alexandria, Egypt \\ ${ }^{3}$ Chemical Engineering Department, Faculty of Engineering, Alexandria, Egypt
}

Email address:

Marwa.f.elkady@gmail.com (M. F. Elkady)

\section{To cite this article:}

M. F. Elkady, M. A. khodary, A. A. Zaatout. Novel Nano-Zirconium Antimonate as Cation Exchange Material for Organic Dye Pollutants Purification. American Journal of Applied Chemistry. Special Issue: Nano-technology for Environmental Aspects.

Vol. 3, No. 3-1, 2015, pp. 46-53. doi: 10.11648/j.ajac.s.2015030301.17

\begin{abstract}
Nano-zirconium antimonate was successfully prepared using two different techniques of sol-gel and homogeneous precipitation. The different preparation parameters affecting onto the two preparation techniques such as $\mathrm{HCl}$ concentration, reactants molar ratio and effect reaction temperature were optimized to attain cation exchange material with high ion exchange capacity and produced in nano-size structure. The different prepared samples were screened based on their ion exchange capacities. The most proper prepared sample produced from the sol-gel technique poses higher ion exchange capacity value of $3.5 \mathrm{meq} / \mathrm{g}$ compared with that produced from the homogeneous precipitation that recorded ion exchange capacity of $2.8 \mathrm{meq} / \mathrm{g}$. The physico-chemical properties of the two nano-zirconium antimonate samples produced from each technique were compared using various analytical techniques such as XRD, SEM and TEM. XRD results indicated that the sample produced from the homogeneous precipitation attains high crystallinity degree compared with the amorphous sol-gel prepared sample. The morphological structure investigation evident the nano-spherical morphology for the two prepared samples. The average nano-size of the homogeneous precipitation sample of $22 \mathrm{~nm}$ is smaller than that of the sol-gel sample that recorded $34 \mathrm{~nm}$. The dye decontamination behavior of the two prepared nano-zirconium antimonate samples was compared for C.I basic blue 3 dye. The sol-gel prepared sample recorded higher dye decolorization of $81.5 \%$ compared with $54 \%$ for the homogeneous precipitation sample.
\end{abstract}

Keywords: Nano- Materials, Zirconium Antimonate, Chemical Characterization, Dye Decolorization

\section{Introduction}

Nano-material is a field that takes a materials science-based approach on nanotechnology. It studies materials with morphological features on the nanoscale, and especially those that have special properties stemming from their nanoscale dimensions. Nanoscale is usually defined as smaller than a one tenth of a micrometer in at least one dimension [1].

Nanotechnology can be defined as the design, synthesis, and application of materials and devices whose size and shape have been engineered at the nanoscale. It exploits unique chemical, physical, electrical, and mechanical properties that emerge when matter is structured at the nanoscale. The physical and chemical properties of nanomaterials can differ significantly from those of the atomic-molecular or the bulk materials of the same composition. Two primary factors cause nanomaterials to behave significantly differently than bulk materials: surface effects that causing smooth properties scaling due to the fraction of atoms at the surface more one, quantum effects (showing discontinuous behavior due to quantum confinement effects in materials with delocalized electrons) [2].These factors affect the chemical reactivity of materials, as well as their mechanical, optical, electric, and magnetic properties. The fraction of the atoms at the surface in nano-particles is increased compared to microparticles or bulk. Accordingly, they have a very large surface area and high 
particle number per unit mass. Different treatment techniques for wastewater polluted with heavy metals have been developed in recent years both to decrease the amount of waste water produced and to improve the quality of the treated effluent. The main techniques, which have been utilized to reduce the heavy metals content of effluents, include chemical-precipitation, adsorption, membrane processes, electrolytic methods and ion-exchange. Ion exchange is an exchange of ions between two electrolytes or between an electrolyte solution and a complex. There are two types of ion exchange materials : organic and inorganic both the organic and inorganic ion exchanger materials can be re-classified as synthetic or natural ion exchange materials according to their resources.

Synthetic inorganic ion exchangers have both thermal resistance and radiation stability and have been applied in reactor chemistry such as reprocessing of irradiated nuclear fuel. This research work going to prepare innovative nanozirconium antimonate using two different techniques. In this respect, the different factors that affect in the properties of the produced Zirconium antimonate will be discussed. Also, physical and chemical characterization of the material will be examined.

\section{Materials and Methods}

\subsection{Reagents and Instrumentation}

The main reagents used for zirconium antimonate preparation using two different techniques are zirconium oxy-chloride (Rankem, Mumbai, India), potassium pyro-antimonate (CHD, New Delhi, India), urea, and hydrochloric acid. All other chemicals and reagents used for properties identification of the prepared zirconium antimonate were of analytical reagent grade. Magnetic stirrer model-526 (J.P.Selecta Co., Spain) was used during the preparation procedure for sol-gel technique and homogenous technique with the aid of identical magnets, An electron microscope (JEOL JSM 6360LA, Japan) has been used for studying morphological properties of the synthesized materials, a spectrophotometer Specord M-42 (Carl Zeiss, Germany) has been used for measuring the concentration of the dye and X-ray diffractometer (Shimadzu-700, USA) was used for determining the $\mathrm{x}$-ray diffraction pattern of the prepared materials.

\subsection{Synthesis of Zirconium Antimonate Using Sol-Gel Precipitation Technique}

A solution composed from potassium pyro-antimonate was added drop wise into a solution of zirconium oxy-chloride with different molar ratio in the presence of hydrochloric acid with constant stirring. After the addition was complete a fine white precipitate appeared. The reaction mixture was diluted to $1 \mathrm{~L}$ and allowed to settle for $24 \mathrm{~h}$ for complete digestion $[3,4]$. The supernatant liquid was decanted and gels were filtered by suction and the excess acid was washed thoroughly with hot water. The washed precipitate was then dried by gentle heating at $60^{\circ} \mathrm{C}$. The effect of hydrochloric acid concentration $(0-0.8 \mathrm{M})$, reaction temperature $\left(25-65^{\circ} \mathrm{C}\right)$ and the molar ratio of reactants $(1: 1,1: 2,1: 4,1: 6,1: 10,2: 1)$ were examined. On the basis of IEC of all prepared samples, one sample will be selected to be characterized.

\subsection{Synthesis of Zirconium Antimonate Using Homogenous Precipitation Technique}

The material was synthesized through the addition of potassium pyro-antimonate solution into zirconium oxy-chloride solution that dissolved in an aqueous hydro-chloric $(0.5 \mathrm{~mol} / \mathrm{L})$ with different molar ratio in presence of $1.5 \mathrm{~g}$ urea. The resulting solution was heated to $90^{\circ} \mathrm{c}$ on an electrical plate with continuous stirring to decompose the precipitating agent. This steps lead to the formation of zirconium antimonate. After precipitation, the material was allowed to rest at $90 \circ \mathrm{C}$ for another hour [5]. The produced precipitate was filtered and washed repeatedly with distilled water for the removal of chloride traces, and then dried at $40 \circ \mathrm{C}$ for $24 \mathrm{~h}$. The effect of urea precipitating agent amounts $(0.5-6 \mathrm{~g})$, reaction temperature $\left(25-65^{\circ} \mathrm{C}\right)$, hydrochloric acid concentration $(0-0.8 \mathrm{M})$ and the effect of the reactants molar ratio $(1: 1,1: 2,1: 4,1: 6,1: 10,2: 1)$ were studied. The largest ion exchange capacity IEC sample will be selected in order to studying its properties.

\subsection{Characterization of the Prepared Nano-Zirconium Antimonate Ion Exchangers}

\subsubsection{Ion Exchange Capacity(IEC)}

The ion exchange capacities of the two different prepared ion exchanger materials were determined by acid-base titration [6]. The weighted sample of the of the prepared material in its $\mathrm{H}^{+}$form were soaked in $25 \mathrm{ml}$ of $2 \mathrm{M} \mathrm{NaCl}$ solution for $24 \mathrm{hr}$ with shaking at ambient temperature to exchange protons with sodium ions. The ion exchanged solution was titrated to the phenolphthalein end point (2 drops of ph.ph indicator, $1 \%$ ph.ph in ethanol) with a $\mathrm{NaOH}$ solution of $0.01 \mathrm{M}$ concentration. The ion exchange capacity (IEC) was calculated using the following equation:

$$
\operatorname{IEC}(\mathrm{meq} / \mathrm{g})=\mathrm{V}_{\mathrm{NaOH}} * \mathrm{C}_{\mathrm{NaOH}} / \mathrm{W}_{\mathrm{d}}
$$

Where, $\mathrm{V}_{\mathrm{NaOH}}, \mathrm{C}_{\mathrm{NaOH}}$ and $\mathrm{W}_{\mathrm{d}}$ are the volume of $\mathrm{NaOH}$ consumed in titration, the concentration of $\mathrm{NaOH}$ solution, and the weight of the dry sample, respectively.

\subsubsection{Effect of Eluant Concentration on IEC}

In order to determine the optimum concentration of the eluant for complete elution of $\mathrm{H}^{+}$ions, $0.1 \mathrm{~g}$ from the prepared material was shaken for $24 \mathrm{~h}$ with $25 \mathrm{ml}$ sodium chloride solution with concentrations $(0.25,0.5,0.75,1,1.5,2 \mathrm{M})$. The elution curves of the hydrogen ion were found for different $\mathrm{NaCl}$ concentrations [7].

\subsubsection{Effect of Contact time on IEC}

0.1 gram of the prepared material was shaken with $25 \mathrm{ml}$ sodium chloride and the amount of librated $\mathrm{H}^{+}$ions was titrated against a standard alkali $\mathrm{NaOH}$ solution after every 1 
hour interval. In order to determine the optimum shaking time of the prepared material with sodium chloride solution for complete elution of $\mathrm{H}^{+}$ions.

\subsubsection{X-ray Diffraction (XRD)}

X-ray powder diffractometry was carried out using X-ray diffractometer (Schimadzu-7000 diffractometer)with $\mathrm{Cu} \mathrm{K \alpha}$ radiation beam $(\lambda=0.154060 \mathrm{~nm})$ in order to determine the structure of the prepared material. The finely powdered samples of the cation exchange materials were packed in to a flat aluminum sample holder, where the X-ray source was a rotating anode operating at $30 \mathrm{kV}$ and $30 \mathrm{~mA}$ with a copper target. Data were collected between $10^{\circ}$ and $80^{\circ}$ in $2 \theta$.

\subsubsection{Scanning Electron Microscope (SEM)}

The prepared material was stocked over aluminum holder and gold-sputtered for examination. The samples were scanned using SEM (JEOL JSM 6360 LA, Japan) to identify the structure of prepared samples and estimate the particle diameter at different magnifications. The average diameter of the prepared material was determined using the software Image tool attached with the equipment.

\subsubsection{Transmission Electron Microscope (TEM)}

A continuous beam of highly accelerated electrons $(30-300 \mathrm{kV})$ is transmitted through a very thin sample $(<$ $100 \mathrm{~nm}$ ) supported on carbon grid at TEM (JEOL JEM-1230, Japan). TEM images are formed from the interaction of the electrons transmitted through the sample. The specimen image generated by the objective lens was subsequently magnified in one or two more magnification stages by the intermediate and projector lens and projected onto a photographic plate.

\subsection{Cation Exchange Behavior of Zirconium Antimonate for Dye Decolorization}

Adsorption tests were performed in a set of Erlenmeyer flasks $(100 \mathrm{ml})$ contains $10 \mathrm{ml}$ of $10 \mathrm{ppm}$ C.I basic 3 dye solutions. $0.1 \mathrm{~g}$ from the different prepared cation exchange material was added to each flask and kept in an isothermal shaker at $25^{\circ} \mathrm{C}$ for 4 hours under agitation at 200rpm to reach equilibrium. The different solid- liquid phases are separated using centrifugation at $6000 \mathrm{rpm}$ for $10 \mathrm{~min}$. The remaining dye concentration in the solutions after sorption process was determined using UV-Vis spectrophotometer M-42 (Carl Zeiss, Germany) at the wavelength $654 \mathrm{~nm}$. The percentage of dye decolorization was estimated using

$$
\% \text { decolorization }=(\mathrm{C} 0-\mathrm{Ce}) / \mathrm{C} 0 \times 100 \%
$$

Where $\mathrm{C} 0$ is the initial dye concentration and $\mathrm{Ce}$ is the final dye concentration at the supernatant at equilibrium.

\section{Result and Discussion}

The effect of the variation the preparation conditions for zirconium antimonate production using each technique onto the ion exchange capacity of the produced material are discussed in details.

\subsection{Zirconium Antimonate Production Using Sol-Gel Technique}

In order to determine the most proper prepared zirconium antimonate, the preparation parameters will be optimized.

\subsubsection{Effect of Hydrochloric Acid Concentration}

Seven samples of zirconium antimonate ion exchanger were prepared using $0.1 \mathrm{M} \mathrm{ZrOCl}_{2}$ and $0.1 \mathrm{M}$ potassium pyro-antimonate at different hydrochloric acid molarities. The ion exchange capacity of these white produced ion exchangers were tabulated in Table1.It was observed that the ion exchange capacity of the produced ion exchanger increased by increasing the molarity of hydrochloric acid. This may be due the increase in the replacement hydrogen ions present inside the exchanger structure, which are responsible mainly for the exchange process inside the exchanger according to the following reaction [8]:

$$
\mathrm{R}-\mathrm{H}+\mathrm{M}^{+} \leftrightarrow \mathrm{R}-\mathrm{M}+\mathrm{H}^{+} \text {(aq.) }
$$

As a result for $\mathrm{HCl}$ concentration, it was observed that the best sample was $\mathrm{S} 6$ which poses higher ion exchange capacity value of $3.5 \mathrm{meq} / \mathrm{g}$.

\subsubsection{Effect of Reactants Molar Ratio}

As mentioned from the previous researches [9] the increment at the anionic part inside the cation exchange material enhances the ion exchange capacity of the prepared material as the replaceable hydrogen ions are attached to this group. So, our study was designed to illustrate the impact of antimonate groups present in zirconium antimonate on IEC that was prepared y using $0.5 \mathrm{M} \mathrm{HCl}$ at different molar ratio. The results presented in Table 1 showed that the increase in antimonate groups increases the IEC of the prepared material. As the amount of zirconium to the antimonate presence at the reaction mixture improved at sample S12 there was no any precipitate formed. This result is in accordance to the suggested formula of zirconium antimonate $\mathrm{Zr}(\mathrm{Sb})_{2}$, where regarding to this formula the amount of antimonates at the reaction mixture may be at least double the amounts of presence zirconium. According to the previous studied factors the best sample that poses the higher IEC was S10. However, the increase at the materials IEC due to the improvement at antimonate was limited. Also, regarding to the economical wise and the rising price of the potassium pyro- antimonate we will select sample S6 as the optimum prepared sample.

\subsubsection{Effect of Reaction Temperature}

The effect of reaction temperature was determined using

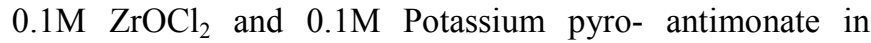
presence of $0.5 \mathrm{M} \mathrm{HCl}$ at different reaction temperatures. Table1 showed a slight increase in IEC of the prepared exchanger as the preparation temperature increased. This may be due to the enhancement of the surface properties of the prepared exchanger caused by the elevation of temperature. Regarding to the reaction temperature has limited enhancement at the material IEC. So, the most proper prepared sample still sample S6 which poses higher ion 
exchange capacity value of $3.5 \mathrm{meq} / \mathrm{g}$.

Table 1. Influence of preparation parameters on the ion-exchange capacity of zirconium antimonate prepared using sol-gel technique

\begin{tabular}{lll}
\hline Sample no. & Preparation parameter & IEC(mequiv/g) \\
\hline HCl concentration(M) & & \\
S1 & 0 & 2.3 \\
S2 & 0.1 & 2.4 \\
S3 & 0.2 & 2.45 \\
S4 & 0.3 & 2.5 \\
S5 & 0.4 & 2.8 \\
S6 & 0.5 & 3.5 \\
S7 & 0.8 & 3.45 \\
Molar ratio & & \\
S6 & $1: 1$ & 3.5 \\
S8 & $1: 2$ & 3.6 \\
S9 & $1: 4$ & 3.65 \\
S10 & $1: 6$ & 3.75 \\
S11 & $1: 10$ & 3.8 \\
S12 & $2: 1$ & No ppt \\
Temperature $\left({ }^{\circ} \mathrm{c}\right)$ & & \\
S6 & 25 & 3.5 \\
S13 & 37 & 3.7 \\
S14 & 45 & 3.75 \\
S15 & 65 & 3.9 \\
\hline
\end{tabular}

\subsection{Zirconium Antimonate Production Using Homogenous Precipitation Technique}

Powdered zirconium antimonate was prepared by homogenous precipitation using urea hydrolysis. The influence of preparation parameters on the IEC of the prepared cation exchanger was examined.

\subsubsection{Effect of Precipitating Agent Amounts}

In order to determine the most proper amount of the precipitating agent (urea) to yield cation exchange material with high IEC, four samples were prepared using $0.1 \mathrm{M}$ $\mathrm{ZrOCl}_{2}$ and $0.1 \mathrm{M}$ potassium pyro-antimonate in presence of $0.5 \mathrm{M} \mathrm{HCl}$ at different amounts of urea. The ion exchange capacity of these produced cation exchangers were tabulated at Table2. It was shown that the ion exchange capacity increases as the amount of urea increases then tends to decrease. This may due to the presence of carbonate precipitate from urea hydrolysis mixed with the produced ion exchanger, which by its role decreased the exchange capacity of the exchanger. It was mentioned before [10] that an excess of precipitating agent should be avoided in the precipitation process in order to avoid contamination of prepared cation exchanger with carbonate, produced from the hydrolysis of urea in an initially acidic solution as follows:

$$
(\mathrm{NH})_{2} \mathrm{CO}+3 \mathrm{H}_{2} \mathrm{O} \rightarrow \mathrm{CO}_{2}+2 \mathrm{NH}_{4}^{+}+2 \mathrm{OH}^{-}
$$

This hydrolytic reaction takes place slowly and occurs at temperature just below the boiling point of water, in order to generate hydroxyl ions, leading to the precipitation of zirconium antimonate with different characteristics [10]. So, as the amount of urea increases the chance for contamination of the prepared exchanger with carbonate increases, which by its role decrease the exchange capacity of the synthesized zirconium antimonate. Accordingly, sample $\mathrm{H} 2$ which poses the higher IEC value of $2.8 \mathrm{meq} / \mathrm{g}$ was selected as the most proper prepared sample.

Table 2. Influence of preparation parameters on the ion-exchange capacity of zirconium antimonate prepared using homogenous precipitation technique

\begin{tabular}{lll}
\hline Sample no. & Preparation parameter & IEC(mequiv/g) \\
\hline \multicolumn{2}{l}{ Precipitating agent amount $(\mathrm{g})$} & \\
H1 & 0.5 & 2.6 \\
H2 & 1.5 & 2.8 \\
H3 & 3 & 2.75 \\
H4 & 6 & 2.5 \\
HCl concentration(M) & & \\
H5 & 0 & 1.9 \\
H6 & 0.1 & 2 \\
H7 & 0.2 & 2.2 \\
H8 & 0.3 & 2.3 \\
H9 & 0.4 & 2.5 \\
H2 & 0.5 & 2.8 \\
H10 & 0.8 & 2.78 \\
Molar ratio & & \\
H2 & $1: 1$ & 2.8 \\
H11 & $1: 2$ & 2.85 \\
H12 & $1: 4$ & 2.9 \\
H13 & $1: 6$ & 3 \\
H14 & $1: 10$ & 3.1 \\
H15 & $2: 1$ & No ppt \\
Temperature( ${ }^{\circ}$ c) & & \\
H2 & 25 & 2.8 \\
H16 & 37 & 2.88 \\
H17 & 45 & 2.9 \\
H18 & 65 & 2.98 \\
\hline
\end{tabular}

\subsubsection{Effect of Hydrochloric Acid Concentration}

The ion exchange capacity of six samples of zirconium antimonate exchanger, using $0.1 \mathrm{M} \mathrm{ZrOCl}_{2}, 0.1 \mathrm{M}$ potassium pyro-antimonate and $1.5 \mathrm{~g}$ urea at different hydrochloric acid molarities. The ion exchange capacity of these produced ion exchangers were tabulated in Table2. It was observed that the ion exchange capacity of the produced ion exchanger increased by increasing the molarity of hydrochloric acid. This may be due the increase in the replacement hydrogen ions present inside the exchanger structure, which are responsible mainly for the exchange process inside the exchanger. The best concentration of $\mathrm{HCl}$ was $0.5 \mathrm{M}$, which poses the higher ion exchange capacity value of $2.8 \mathrm{meq} / \mathrm{g}$.

\subsubsection{Effect of Reactants Molar Ratio}

From the previous studied preparation factors; it was observed that the effect of molar ratio could be investigated through zirconium antimonate preparation using different molar ratio in presence of $0.5 \mathrm{M} \mathrm{HCl}$ and $1.5 \mathrm{~g}$ urea. It was observed from Table2 that the increase at the antimonate groups at the material increases its IEC this may be returned to the responsibility of the antimonate group to the $\mathrm{H}^{+}$ exchanging inside the exchanger. However, the improvement at the ratio of zirconium to the antimonate there was no any precipitate formed as evident at the sol-gel precipitation technique. From these results, the best sample which poses the higher IEC was H14. However, the increment at the material IEC was limited compared with the improvement at the antimonate group. So, regarding to the rising price of the potassium pyro-antimonate we will consider sample $\mathrm{H} 2$ as the 
most proper prepared sample [12].

\subsubsection{Effect of Precipitating Temperature}

The IEC for zirconium antimonate cation exchange material that precipitated at various temperatures was studied using $0.1 \mathrm{M} \mathrm{ZrOCl}_{2}, 0.1 \mathrm{M}$ potassium pyro-antimonate, $0.5 \mathrm{M}$ $\mathrm{HCl}$ and $1.5 \mathrm{~g}$ urea. it is clear from the results that the value of IEC material increased with increases the temperature, regarding the hydrolytic reaction of urea which takes place slowly and occurs at temperatures just below the boiling point of water, this result is in accordance with the fact that the decomposition rate of urea is strongly depends on the temperature [11]. The effect of precipitating temperature has limited increase in the IEC of the cation exchanger. So, the selected sample from this studied factor was $\mathrm{H} 2$ which poses higher ion exchange capacity value of $2.8 \mathrm{meq} / \mathrm{g}$.

Finally from the previously studies preparation parameters using sol-gel precipitation and homogenous precipitation techniques samples S6\&H2 was selected as the most proper prepared samples produced from each technique respectively.

\subsection{Characterization of the Synthesized Cation Exchange Materials}

The selected samples were chemically characterized; first we determine the optimum eluant concentration and eluant time in order to obtain the most precision results.

\subsubsection{Effect of Eluant Concentration and Elution Time on Ion Exchange Capacity}

The elution of zirconium antimonate ion exchanger is governed by both eluant concentration and elution time, which was found as a usual behavior for these types of inorganic ion-exchange materials. The minimum molar concentration of $\mathrm{NaCl}$ as eluant and the minimum elution times for zirconium antimonate samples prepared using sol-gel precipitation and homogenous precipitation were found to be $1.5 \mathrm{M}, 15 \mathrm{~h}$ and $1 \mathrm{M}, 15 \mathrm{~h}$ respectively for maximum release of $\mathrm{H}^{+}$ions from $0.1 \mathrm{~g}$ of the cation-exchangers as shown from figures $(1 \& 2)$. As a result, the exchange capacity of the selected samples was determined using $1.5 \mathrm{M}$ salt solution and samples are equilibrated for $24 \mathrm{~h}$ to confirm that all $\mathrm{H}^{+}$ions are released from zirconium antimonate.

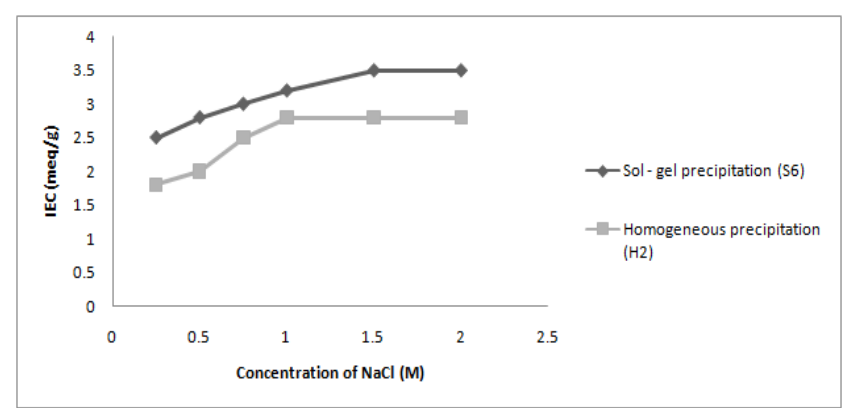

Figure 1. Effect of $\mathrm{NaCl}$ concentration on zirconium antimonate ion exchange capacity

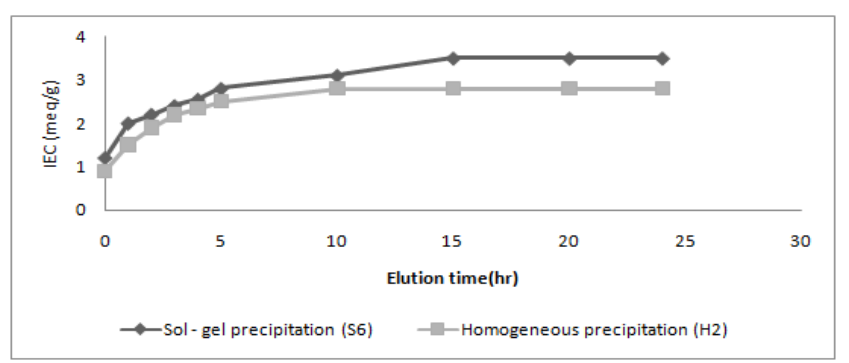

Figure 2. Effect of elution time on zirconium antimonate ion exchange capacity

\subsubsection{Crystalline Structures of Prepared Zirconium Antimonates}

The X-ray diffraction patterns of the two selected samples (S6 and H2) were investigated at figures $(3,4)$. The X-ray diffraction spectrum showed a number of peaks at different $2 \theta$ values. The strong peaks intensities of sample $\mathrm{H} 2$ compared with sample S6 suggested that the sample produced from the homogeneous precipitation attains high crystallinity degree compared with the amorphous sol-gel prepared sample. The different characterstics peaks presented in each sample were compared with two references patterns of zirconium oxide and antimony oxide samples. It was indicated that the characteristics peaks at $2 \theta=28.2^{\circ}$ and $31.5^{\circ}$ are representative to zirconium oxide. However the characteristic peak at $2 \theta=$ $30^{\circ}$ is representative to antimony oxide. Accordingly, it was clear from the previous data that the produced sample represents a mixture of zirconium oxide and antimony oxide [13].

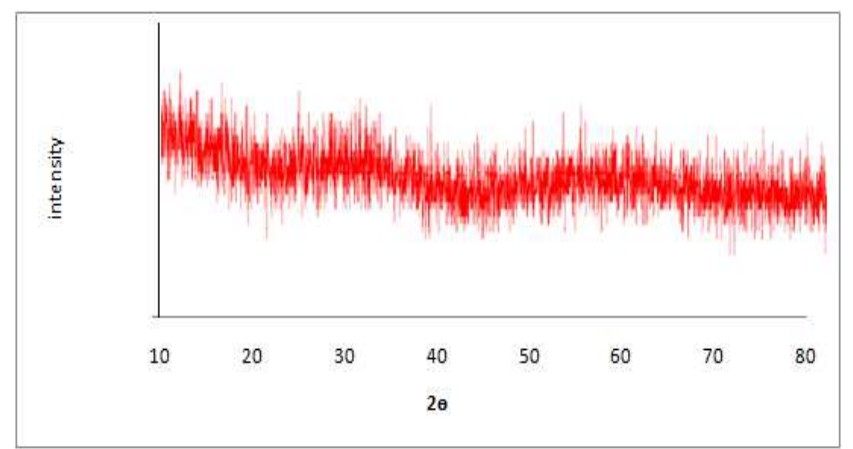

Figure 3. X-ray diffraction of zirconium antimonite prepared using sol-gel technique

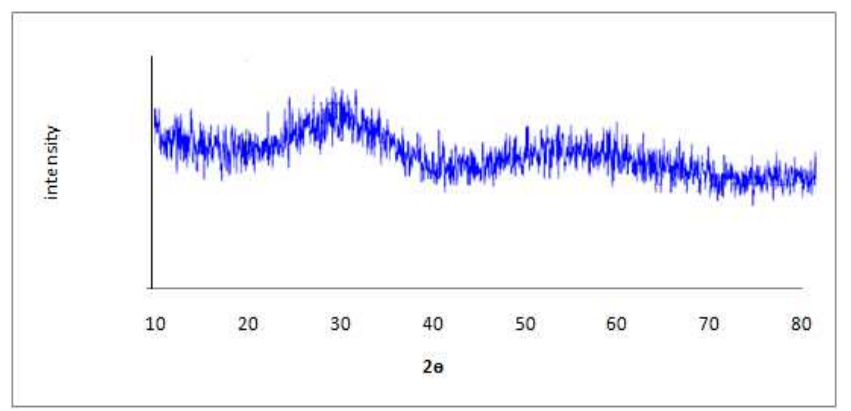

Figure 4. X-ray diffraction of zirconium antimonate prepared using homogeneous precipitation technique 


\subsubsection{Morphological Structure of Prepared Zirconium Antimonates}

Morphological characterization of the prepared cation exchanger zirconium antimonate samples produced from each preparation technique was performed using both SEM and TEM. It was evident from SEM and TEM imaging that the prepared cation exchange material either that produced from sol-gel or homogenous precipitation techniques were prepared in spherical particles morphological structures. Figure (5) indicates that the average particle size of sample S6 from the sol-gel precipitation technique equal to $34 \mathrm{~nm}$, which is comparatively larger than that produced from the homogenous technique $\mathrm{H} 2$ that has $22 \mathrm{~nm}$ average particle sizes. However, figure (6) illustrated that the average particle diameter of the sol-gel prepared sample is $12 \mathrm{~nm}$ compared with $10 \mathrm{~nm}$ for the homogeneous precipitated technique. The large nano-size investigated through the SEM imaging may be regarded to the nano-particles aggregates. Accordingly from the SEM and TEM imaging, the nano-size of the prepared zirconium antimonite samples was confirmed.

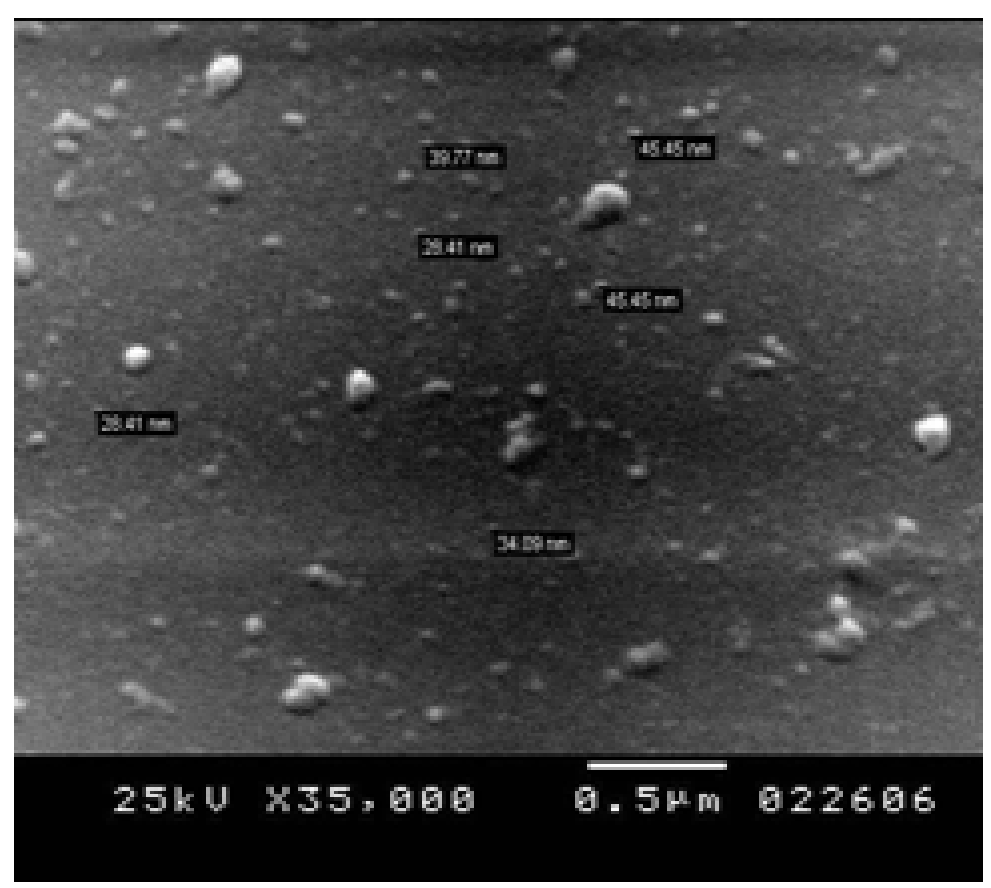

(a)

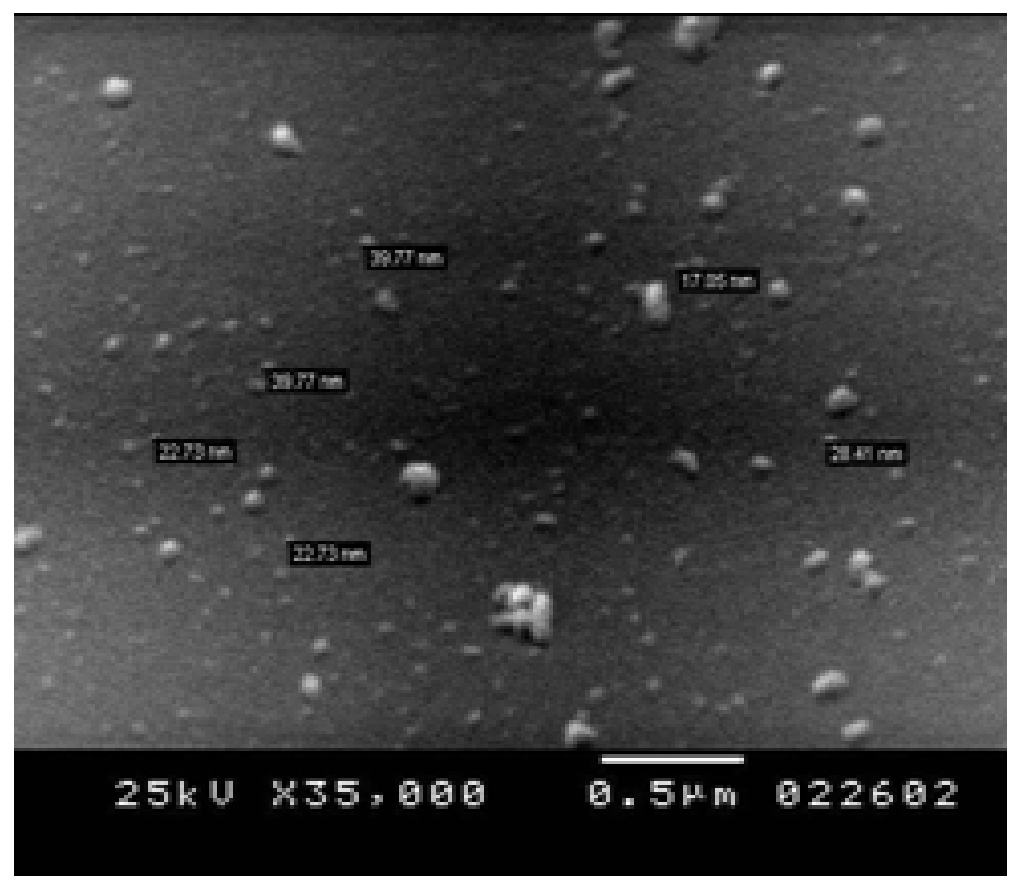

(b)

Figure 5. Scanning electron microphotographs of zirconium antimonate produced from (a) sol-gel preparation, (b) homogenous precipitation. 


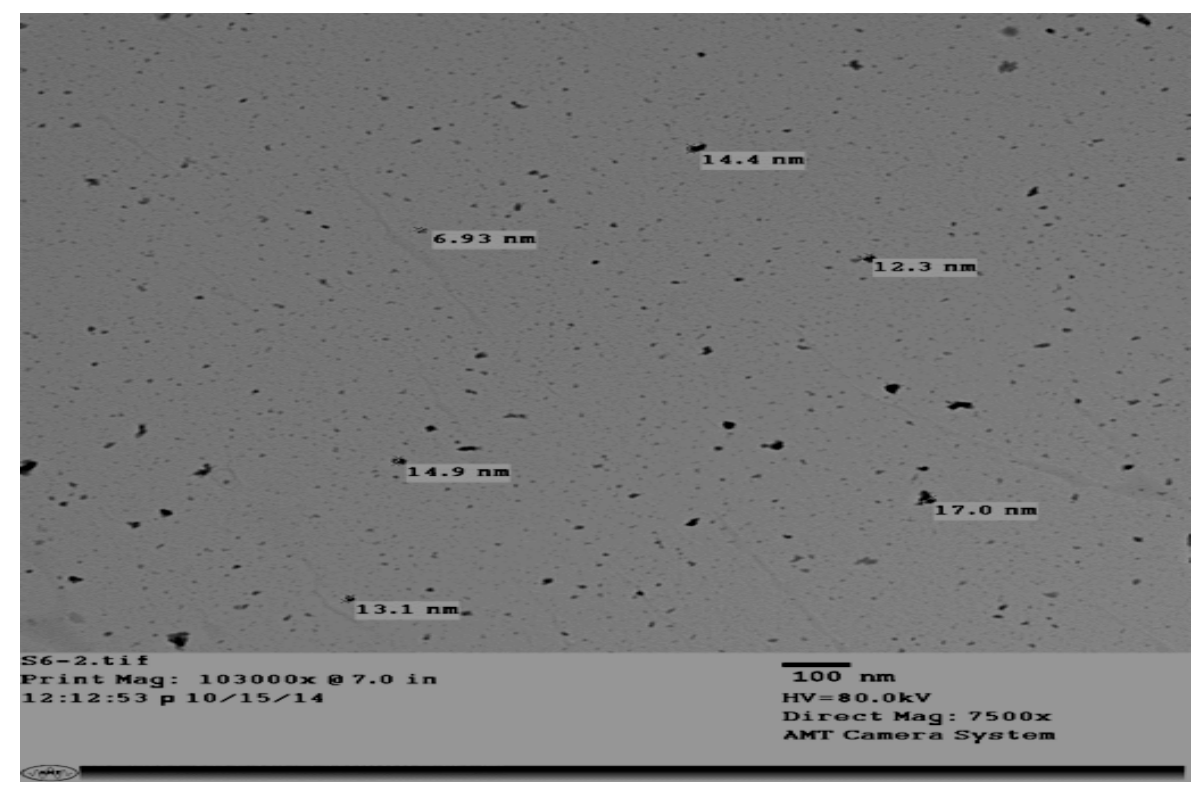

(a)

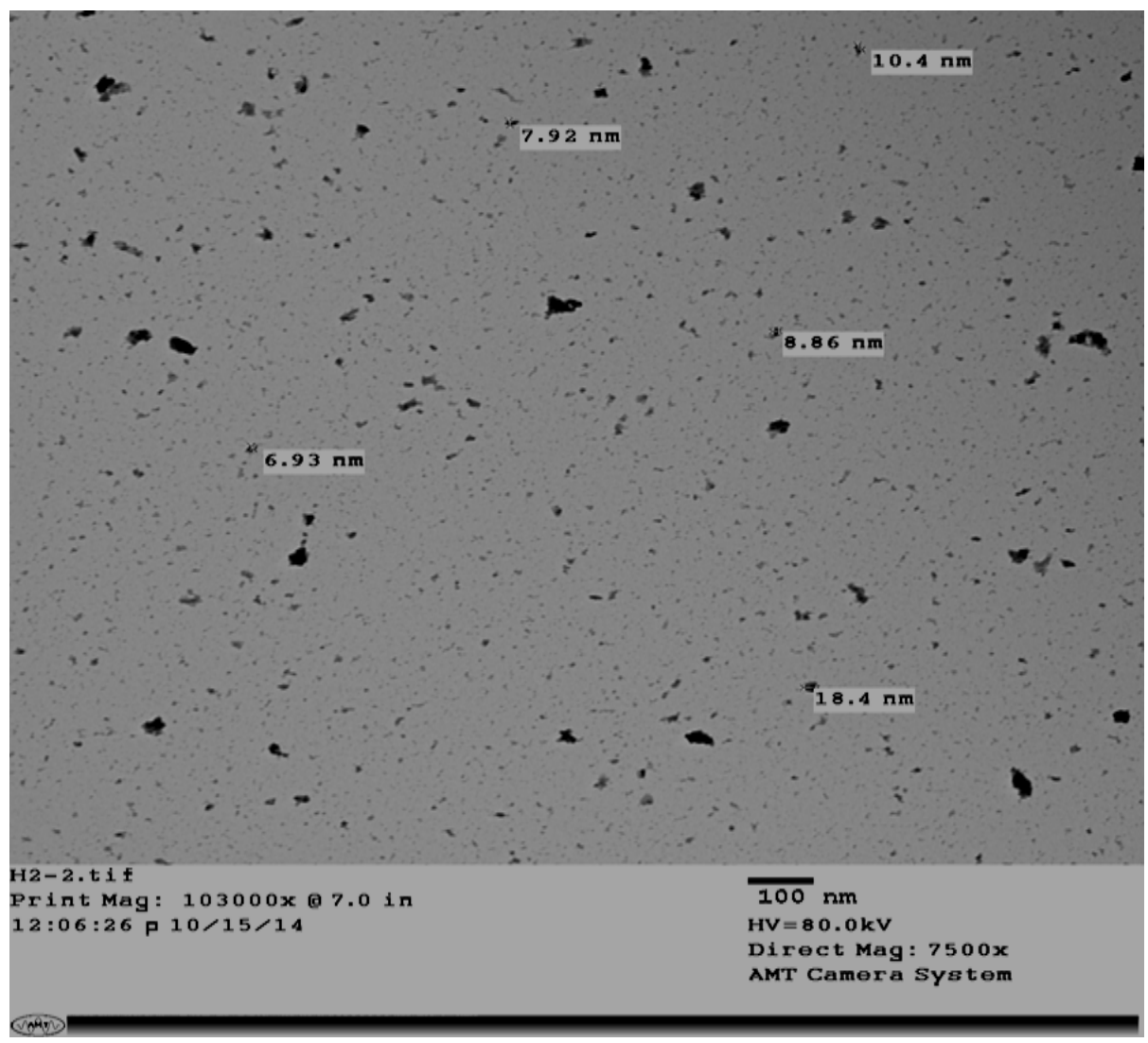

(b)

Figure 6. Transmission electron microscopy of zirconium antimonate produced from (a) sol-gel preparation, (b) homogenous precipitation.

\subsection{Cation Exchange Behavior of Zirconium Antimonate for Dye Decolorization}

In order to determine the exchange efficiency of the prepared material for cationic dye ions, the dye decontamination behaviors of the most proper two prepared nano-zirconium antimonate samples produced from sol-gel and homogeneous precipitation techniques was compared toward C.I basic blue 3dye. It was indicated from figure (7) that the sol-gel prepared sample recorded higher dye decolorization of $81.5 \%$ compared with $54 \%$ for the homogeneous precipitated sample. These dye decolorizations results confirm the ion exchange capacity results, where it was indicated that the sol-gel prepared sample possess higher IEC and decolorization efficiency compared with the 
homogeneous precipitated sample [14].

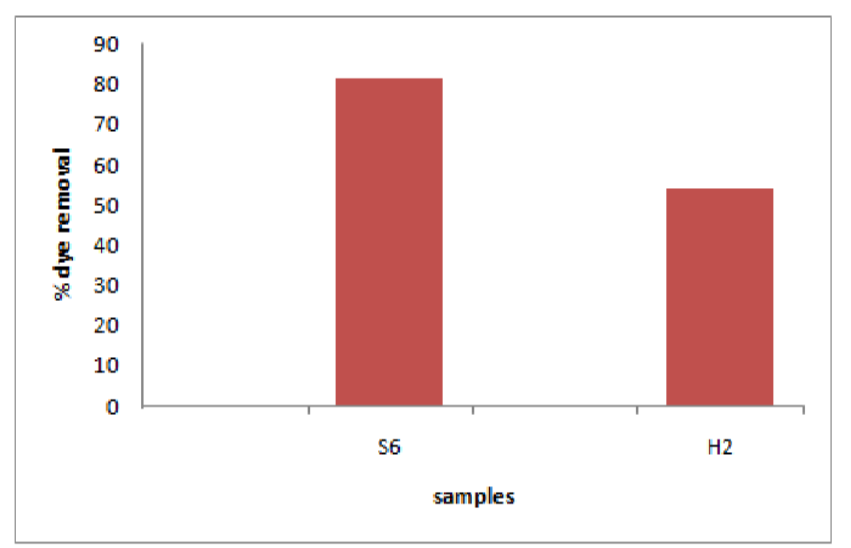

Figure 7. \% C.I basic blue 3dye sorption using the two different prepared samples produced from sol-gel technique and homogenous precipitation technique.

\section{Conclusion}

Two different preparation techniques sol-gel precipitation and homogeneous precipitation are used for the preparation of a novel nano-zirconium antimonate with appropriate ion exchange properties. The ion exchange properties of the synthesized materials were controlled by systematic changes in the synthetic variables of each preparation technique, in order to improve the properties of the exchangers and their performance. The most proper sol-gel prepared ion exchange material was produced from the reaction of $0.1 \mathrm{M} \mathrm{ZrOCl}_{2}$ with $0.1 \mathrm{M}$ potassium pyro-antimonate in presence of $0.5 \mathrm{M} \mathrm{HCl}$ at $25{ }^{\circ} \mathrm{C}$. This sample recorded the highest IEC value of 3.5 meq/g compared with the other homogeneous precipitated sample. The ion exchange capacity of the most proper homogeneous precipitated sample recorded as $2.8 \mathrm{meq} / \mathrm{g}$. this sample produced from the reaction of $0.1 \mathrm{M} \mathrm{ZrOCl}_{2}$ and $0.1 \mathrm{M}$ potassium pyro-antimonate in presence of $1.5 \mathrm{~g}$ urea and 0.5 $\mathrm{M} \mathrm{HCl}$ at $25{ }^{\circ} \mathrm{C}$. The XRD spectrum of these two samples indicated that the homogeneous precipitated sample is more crystalline compared with the sol-gel prepared sample. SEM and TEM imaging confirms the nanostructure of the two prepared samples. The dye decontamination behavior of the two prepared nano-zirconium antimonate samples was compared toward C.I basic blue 3dye. It was indicated that the sol-gel prepared sample recorded higher dye decolorization of $81.5 \%$ compared with $54 \%$ for the homogeneous precipitated sample.

\section{References}

[1] M.M. Abd El-Latif, M.F. Elkady, Materials Research Bulletin, 46 (2011) 105-118.

[2] M. F. Elkady, E. M. El-Sayed, H. A. Farag, A. A. Zaatout, Journal of Nanomaterials, 1 (2014) 1-11.

[3] S.Lahiri,K.Roy,S.Bhattachary,S.Maji,S.Basu, Appl.Radiat.Isot. 63(2005)293-297.

[4] K.Roy,S. Basu, A.Ramaswami, S. Lahiri, Appl. Radiat. Isot., 59(2003) 105-108.

[5] H. Shokry Hassan, M.F. Elkady, Ahmed El-Shazly, Hesham Bamufleh, Journal of Nanomaterials, 1 (2014) 1-14.

[6] M. Rikukawa, D. Inagaki, K. Kaneko, Y. Takeoke, I. Ito, Y. Kanzaki, K. Sanui, J. Mol.Stuct., 739 (2005) 153-161.

[7] Y. Inoue, J. Inorg. Nucl., Chem., 26 (1964) 2241-2253.

[8] C. Abraham, Inorganic Ion Exchange Materials, CRC Press Inc., Boca Roton, Fl, 1982.

[9] S.A. Nabi, M. Naushad, Inamuddin, J. Hazard. Master., 142 (2007) 404-411.

[10] K. Roya, P.K. Mohapatrab, N. Rawatb, D.K. Pala, S. Basua, V.K. Manchanda, Appl. Radiat. Isot., 60 (2004) 621-624.

[11] M. F. Elkady, M. M. Hussein, M. M. Salama, American Journal of Applied Chemistry, 3 (2015) 1-7.

[12] W.H.R.Shaw, J.J.Bordeaux, J.Am.Chem.Soc.,77 4729-4733.

[13] G. Socrates, Infrared Characteristic Group Frequencies, John Wiley \& Sons Ltd., New York, 1980.

[14] M.F.Elkady, Mohamed Hussien, Reham Abou-rady, Bothalia Journal, 44 (2014) 115-138. 